permanent magnets; but, as regards the understanding of fundamental physical phenomena, magnetism appears to have played a part similar to that of money in economics - a useful link for facilitating exchange, but a source of unnecessary complexity and confusion in discussion. Since we can formulate the forces acting on moving electric charges directly from Ampère's experiments without introducing magnetism, and thereby dispense with the two stages of reasoning involved in Maxwell's curl equations, it seems decidedly advantageous to do so; and if a magnetic field is merely a translationally moving electric field, as the flux-cutting principle suggests, it seems superfluous to regard it as a separate entity.

What we have hitherto called an electromagnetic wave is simply a wave of electric force and displace. ment, or electric wave, corresponding to the pressure and displacement in an acoustic wave; and the dualistic concept of two waves of electric and magnetic force in perpendicular planes is a superfluous complication. The electrostatic forces between stationary charged bodies and the propagation of electric waves can be illustrated satisfactorily by assuming protons and electrons to be connected by contractile tentacles or bonds, and the only phenomenon which seems unexplainable by mechanical principles is the transverse force on a moving charge caused by the proximity of another moving charge. This is perhaps the most important fundamental problem in physics and may seem to justify the vortical concept of a magnetic field; but this concept does not appear to provide a convincing explanation. We do know, however, from Ampère's experiments, what this force is ; and the general formula given in my letter in NATURE of December 3 enables us clearly to understand the behaviour even of dynamos and transformers without any consideration of magnetism.

Mr. Appleyard has, however, raised another important fundamental point by directing attention to the fact that this force as given by the second

term in my equation : $E_{2}=\frac{e}{c^{2} r^{3}}\left[V^{\prime}\left[V_{\mathrm{P}}\right]\right]$, has no

meaning unless the velocities $V$ and $V^{\prime}$ are defined with respect to a basic framework or medium. This term is in accordance with Maxwell's treatment of moving charged surfaces ("Treatise", 2, xix, \& 769); with Ampère's experiments; and with the fluxcutting equations given in my article in NATURE of February 5, 1938. These equations : $H^{\prime}=[V J]=$ $\chi[V E]$, and $E^{\prime}=-[V B]=-\mu[V H]$, appear to be the true fundamental equations if the magnetic link is employed, and lead to the relation $E^{\prime}=x \mu V^{2} E$ for an isotropic dielectric medium if that link is eliminated; but it is evident that they also have no meaning for space unless $V$ is defined relatively to a basic framework or medium; and the physical picture of electric wave propagation which arises from them is so clear and convincing as to leave no room for doubt that electric waves, including light, are undulatory electric displacements in a basic medium or ether which has the properties of a dielectric. I believe that this will be acknowledged in due course, and that the classic theories and concepts of Newton, Faraday and Maxwell will be restored to their rightful eminence.

The Athenæum.

C. V. Drysdale.

Jan. 5.
Ultra-violet Absorption Spectrum and Chemical Reactivity of some Substituted 9-Vinyl-Phenanthrenes

THE direct conjugation between two aromatic nuclei or between an aromatic nucleus and an olefinic double bond expresses itself in the spectrum : diffuse bands appear instead of the sharp ones and at higher wave-lengths (diphenyl ${ }^{1}$, di- $\alpha$-naphthyl ${ }^{2}$, styren $\theta^{3}$, etc.). It is therefore worth reporting that $9,9^{\prime}$ diphenanthryl (I), although having a system of two conjugated olefinic double bonds, exhibits a spectrum which is practically identical with that of phenanthrene $e^{4}$ (two regions of absorption: persistent, diffuse bands between 2700 and $3000 \mathrm{~A}$., and sharp bands at higher concentrations, between 3000 and 3500 A.). The same is true for 9-cyclohexenylphenanthrene (II), which does not behave spectroscopically analogously to styrene.

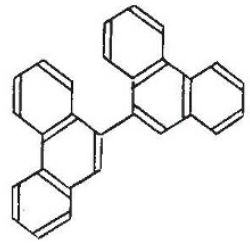

I

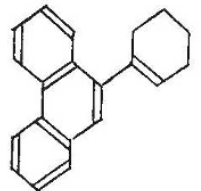

II

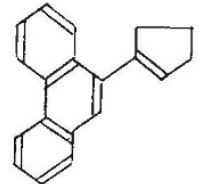

III
It is interesting that the two hydrocarbons, according to $\mathrm{E}$. and $\mathrm{F}$. Bergmann ${ }^{5}$, do not show any 'dienic' activity, for example, towards maleic anhydride. If there really is a parallel between this inactivity and the spectral behaviour, one would expect that for a substance analogous to (II), but having an active conjugation, the spectrum should show the above-quoted characteristic features. This is actually the case : 9-cyclopentenyl-phenanthrene (III), which easily combines with maleic anhydride, has fower and markedly more diffuse bands than phenanthrene, at the same time shifted towards the visible part of the spectrum.

A detailed description of our results and a report on other cases of such an unusual coincidence will be given elsewhere.

Institute of Physical Chemistry, VICTOR HENRI. University, Liège.

Daniel Sieff Research Institute,

Ernst Bergmann. Rehovoth, Palestine. Dec. 25.

1 Pestemer and Mayer-Pitsch, Monatshefte, 70, 104 (1937). Titeica, C.R. Acad. Sci., 199, 458 (1934).

${ }^{2}$ Pestemer and Cecelsky, Monatshefte, 58, 113 (1932). Brass and Patzelt, Ber, deutsch. Chem. Ges., 70, 1349 (1937).

${ }^{3}$ Ley and v. Engelhardt, $Z$. phys. Chem., 74, 31 (1910). Ley and Dirking Ber, deutsch Chem Ges., 67, 1331 (1934). Pestemer Dirking, Ber, deutsch. Chem. Ges., 67, 1331

4 Mayneord and Roe, Proc. Roy. Soc., A, 152, 299 (1935). Godart, Bull. Roy. Soc. des Sciences liège, No. 3-4 (1938)

${ }^{5}$ Bergmann, E., and Bergmann, F., J. Amer. Chem. Soc., 59, 1443 (1937).

\section{Atomic Lines in the Auroral Spectrum}

Prof. L. VEgard's recent note ${ }^{1}$ on the atomic lines in the auroral spectrum calls for some comments. While it is true that the presence of the Vegard-Kaplan bands in auroral spectra was first observed and interpreted by Vegard, R. Bernard reported an auroral spectrum in which these bands 